\title{
In Depth Analysis of Clotting Dynamics in Burn Patients
}

Shawn Tejiram, MD ${ }^{\mathrm{a}, \mathrm{b}}$, Kathleen E. Brummel-Ziedins, $\mathrm{PhD}^{\mathrm{c}}$, Thomas Orfeo, $\mathrm{PhD}^{\mathrm{c}}$, Kenneth G. Mann, $\mathrm{PhD}^{\mathrm{c}}$, Brittany N. Hamilton, $\mathrm{BS}^{\mathrm{b}}$, Lauren T. Moffatt, $\mathrm{PhD}^{\mathrm{b}}$, Russell P. Tracy, $P h D^{d}$, and Jeffrey W. Shupp, MD ${ }^{a, b}$

${ }^{a}$ The Burn Center, Department of Surgery, MedStar Washington Hospital Center, Washington, DC

${ }^{\text {b }}$ Firefighters' Burn and Surgical Research Laboratory, MedStar Health Research Institute, Washington, DC

${ }^{\mathrm{C}}$ Department of Biochemistry, University of Vermont, Colchester, VT

d Department of Pathology and Laboratory Medicine, University of Vermont, Colchester, VT

\section{Corresponding Author:}

Jeffrey W. Shupp, MD

\author{
The Burn Center \\ Department of Surgery \\ MedStar Washington Hospital Center \\ Room 3B-55 \\ 110 Irving Street, NW \\ Washington, DC 20010-2975 \\ Phone: 202-877-7347 \\ Fax: $\quad 202-877-7302$ \\ jeffrey.w.shupp@medstar.net
}

Conflict of Interest: Kenneth G. Mann, PhD -- Chair of the Board of Directors for Haematologic Technologies, Inc.

Funding Source: Department of Defense, Department of the Army, Grant/Contract Numbers: W911NF-10-1-0459, Systems Biology Program ARO-W911NF-10-1-0376, and NIH-DOD TACTIC study 1-UM-1-HL120877-1, project 6.

Author Contributions: All authors have made equal contributions to the conception, design, analysis, interpretation, drafting, and revision of the manuscript for this study.

Category: Shock/Sepsis/Trauma/Critical Care

Keywords: Coagulopathy, INR, Clotting Factor, Burn Injury, Thermal Injury 


\section{Abstract}

Background: Studies associating coagulopathic changes with burn injury have relied on limited tests such as PTT and INR. Understanding the clotting dynamics and associated risk factors following burn injury could influence management. This work aimed to identify real time changes in coagulation following burn injury not indicated by PTT or INR alone.

Materials and Methods: Nine burn injured patients at a regional burn center were enrolled for blood collection at admission and set intervals over 96 hours. Patient demographics, management, and laboratory data (PTT and INR) were collected. Plasma assays determined factors II, V, VII, VIII, IX, X, XI, antithrombin, and protein $\mathrm{C}$ functional activity as well as PAP, D-Dimer, fibrin monomer, TFPI, IL-1b, IL-6, IL-10, IL-12p.70, and TNF- $\alpha$ concentrations.

Results: Overall, five patients died. These patients had higher mortality scores and were more acidotic. All patients had normal coagulation studies (INR $<1.5$, PTT<45s) within 24 hours of admission and only two were abnormal after. Increased factor VIII and IX activity were identified in seven patients at admission. Decreased antithrombin and protein $\mathrm{C}$ activity were seen in all patients. Patients had increased PAP, D-Dimer, and fibrin monomer concentrations throughout their hospital course. At admission, increased fold changes were seen in IL-6 (2.5-117) and IL-10 (2.4-32) while IL-1b and TNF- $\alpha$ levels were depressed in all patients.

Conclusion: Extensive changes not identified by PTT or INR were seen following burn injury that may explain perturbed coagulation in these patients. This approach further characterizes the impact thermal injury has on coagulation. 


\section{Introduction}

Major homeostatic changes in coagulation have been described in the trauma literature following a multifactorial process initiated by hemorrhagic shock, endothelial injury, factor consumption, hypothermia, acidosis and more [1]. These processes impair the interactions of procoagulants, anticoagulants, and fibrinolytic components that are crucial for proper hemostasis [2]. The acute traumatic coagulopathy (ATC) that can occur after injury results in increased transfusion requirements, injury severity scores, organ dysfunction and overall mortality [2-4]. Data from the Center for Disease Control noted that of 180,000 deaths in 2010, most fatal sequelae of traumatic injury were attributed to hemorrhagic shock and multiple organ failure [5]. Treatment strategies to manage ATC and improve outcomes have since developed. US military studies, for example, have shown improved outcomes with administration of fresh whole blood or plasma compared to the usage of red cell concentrates [6].

A growing body of literature has similarly identified coagulopathic changes in patients following burn injury. Though sometimes considered a subset of the trauma population, burn injury is inherently different. In trauma patients, severe tissue injury and systemic hypoperfusion due to hemorrhage are major contributors to ATC. Burn injured patients in comparison do not experience the significant hemorrhagic injuries or massive blood transfusions that often characterize the trauma population [7]. Clinical metrics such as burn size, temperature, number of procedures, inhalation injury, and mechanical ventilation are all thought to factor proportionally into coagulation behavior [7-12]. Molecular 
changes, such as inflammatory system activation, as well as clinical management with burn wound excision can extend or even worsen the negative impact on the coagulation system [13]. Septic complications can further drive thrombosis, leading to cellular hypoperfusion, multi-system organ failure, or even death [13].

Current routine measures of coagulation include laboratory assessments such as prothrombin time (PT), partial thromboplastin time (PTT), and the international normalized ratio (INR) [1]. The ubiquity and availability of these tests allow clinicians to assess the integrity of the intrinsic and extrinsic coagulation pathways in these patients. However, it takes some time for these tests to be performed and they only provide a limited snapshot of a patient's overall coagulation profile [14]. For example, these tests are insensitive to alterations in both the status of the protein $\mathrm{C}$ system and the fibrinolytic system. Systemic anticoagulation via protein $\mathrm{C}$ activation has been proposed as a mechanism of ATC as a hyperfibrinolyitic state [2]. Further changes in coagulation can manifest later due to factor consumption, dilution, or delayed fibrinolysis and may be missed by standard laboratory testing. Continued clinical intervention in burned patients, such as central venous access, mechanical ventilator management, or fluid administration can similarly cause delayed coagulation changes $[15,16]$. This can significantly delay identification and treatment of coagulopathy in a thermally injured patient.

Coagulopathy has been identified as a risk factor for increased morbidity and mortality [17].Understanding the pathophysiology and timing behind 
coagulopathy in burn injured patients has major implications and could lead to better transfusion and blood loss management at admission, later in the hospital course, and following burn wound excision. Previous studies examining coagulation changes in thermally injured patients are typically retrospective in nature and rely on routine measures of coagulation. Measurements are usually infrequent and don't account for the rapid and dynamic nature of coagulation itself following injury. If coagulation abnormalities following thermal injury could be better characterized, the potential for new or improved therapeutic intervention could arise.

The aim of this study was to develop a real time assessment model to examine and characterize acute changes and associated risk factors in the coagulation profile of thermally injured patients and compare those changes to established routine measures of coagulation. 


\section{Methods}

\subsection{Setting}

The Burn Center at MedStar Washington Hospital Center is a regional urban burn center providing total burn care to the Washington metropolitan area, serving Washington, D.C., southern Maryland, northern Virginia, and eastern West Virginia. MedStar Washington Hospital Center is a 926 bed Level I Trauma Center. The Burn Center is equipped with an eighteen bed burn rehabilitation and intermediate care unit as well as a dedicated burn ICU and operating suite.

\subsection{Patients}

Following IRB approval, nine thermally injured patients with a total body surface area (TBSA) burn size of greater than or equal to $25 \%$ were identified upon presentation to the trauma bay between 2013 and 2014 and enrolled for study after informed consent. Patients who presented with chemical burn injuries, were under 18 years of age, actively taking anticoagulants, not fluent in either English or Spanish, presented more than four hours after injury, or were otherwise thought not fit for inclusion based on pre-existing conditions were not included for study.

\subsection{Study Design}

Following consent, standardized time points for blood collection were scheduled at admission and at hours 2, 4, 8, 12, 24 and every 12 hours after until 96 hours (4 days) after admission. Samples were collected in SCAT-144 tubes (500 $\mu \mathrm{M}$ AEBSF, $20 \mu \mathrm{M}$ elastinal, $10 \mu \mathrm{M}$ GGACK, 4.5 mM EDTA, $5 \mu \mathrm{M}$ E64, 1 $\mu \mathrm{M}$ Repastin A, $300 \mathrm{KIU} / \mathrm{ml}$ aprotinin; Haematologic Technologies, Inc., Essex Junction, VT) and citrated tubes for further analysis. Plasma obtained from these 
samples was then used to determine clotting factor activity, natural anticoagulant activity, and fibrinolytic and cytokine concentrations for all time points. Routine measures of coagulation, including partial thromboplastin time (PTT) and the international normalized ratio (INR), were obtained at the discretion of the managing clinical team. Patients were grouped by burn size and mortality to identify trends and differences. Burn size was grouped as low (25-50\% TBSA), moderate $(50-75 \%$ TBSA), and severe ( $>75 \%$ TBSA). No study data was used for clinical decision making and treating clinicians were blinded to the study results.

\subsection{Plasma Assays}

Coagulation assays assessing the functional activity of factors II, V, VII, VIII, IX, X and XI, antithrombin and protein C. were performed by the University of Vermont Medical Center Clinical Laboratory (Burlington, VT) using citrate plasma samples. ELISA based assays on SCAT-144 plasmas were used to determine the concentrations of tissue factor pathway inhibitor (Asserachrom Total TFPI, Diagnostica Stago S.A.S, France), plasmin-antiplasmin complex (Imunoclone PAP, Sekisui LLC, USA), d-dimer (LIATEST D-DI, Diagnostica Stago S.A.S, France) and fibrin monomer (LIATEST FM, Diagnostica Stago S.A.S, France). Assays of inflammatory markers in SCAT-144 plasmas were performed by the Laboratory for Clinical Biochemistry Research at the University of Vermont. IL-1b, IL-6, IL-10 and IL-12p.70 were measured using the Proinflammatory panel 1 V-Plex plus plate (Meso Scale Diagnostics LLC, USA). 
TNF- $\alpha$ was measured using the Millipore CVD3 multiplex panel (EMD Millipore, USA).

\subsection{Clinical Measurements and Parameters}

Electronic medical records and paper charts were reviewed during the study period for data on demographics, injury characteristics, clinical management, and outcomes. Abnormal coagulation as determined from routine clinical laboratory measures was defined as an INR $>1.5$ or PTT $>45$ seconds. Additional laboratory parameters recorded included standard vital signs and measures of acidosis (blood $\mathrm{pH}$, base deficit, and serum $\mathrm{CO}_{2}$ ) to evaluate for signs of the lethal triad. Severity of illness was determined using mortality scores, including the Baux and FLAMES (Fatality by Longevity, APACHE II score, Measured Extent of burn, and Sex) scoring [18].

\subsection{Statistical Analysis}

Data was stored and collated using Microsoft Excel (Microsoft, Redmond, WA). Data analysis was performed using GraphPad (GraphPad Software, Inc, La

Jolla, CA). Data is reported as the mean with standard deviation. Statistical comparisons were made using student's t-test and ANOVA with a statistical significance set at $p<0.05$. 


\section{Results}

\subsection{Demographics}

Nine patients were admitted for burn related critical care management and enrolled for study. TBSA burn size ranged from $25 \%$ to $95 \%$. Three patients were grouped into the low burn size group (TBSA 25-50\%), two into the moderate burn size group (TBSA $50-75 \%$ ), and four into the severely burn sized group (TBSA > 75\%). Eight patients were male and one was female. All burns were flame related. Eight patients required mechanical ventilation during the study period. Comorbidities included hypertension, diabetes, schizophrenia, depression, alcoholism, arthritis, diverticulitis, hepatitis C, and myocardial infarction. Average age was $49.4 \pm 16.6$ and ranged from 26 to 77 years. The mean time between burn injury to presentation was $95.6 \pm 55.5$ minutes.

\subsection{Clinical Data and Mortality}

Overall, five patients died and four patients survived. Patients who died had significantly higher Baux (125.26 vs 91.05, $p<0.05$; Figure 1) and FLAMES $(60.57 \%$ vs $32.4 \%, p<0.05$; Figure 1) scores than patients who survived. Mortality patients also had a higher number of inhalation injuries (5 vs 3 ) and received a higher number packed red blood cells (13 vs 6 ), fresh frozen plasma (36 vs 24), and platelet (4 vs 1) transfusions during the 96 hour period.

The acid-base status of patients was determined using blood $\mathrm{pH}$, base deficit, and serum $\mathrm{CO}_{2}$. By all measures, each showed worse and prolonged periods of acidosis in patients who died compared to those who survived (Figure 2). Although patients who survived experienced decreased blood $\mathrm{pH}$, base 
deficits, and serum $\mathrm{CO}_{2}$ within the initial 12 hours period following admission, they recovered shortly after with no subsequent abnormalities.

Both survival and mortality group mean temperature was hypothermic $(T<$ $35^{\circ} \mathrm{C}$ ) in the initial 24 hours following admission, but recovered afterwards without further temperature related issues.

\subsection{Routine Measures of Coagulation}

Only two patients were individually identified as having abnormal coagulation by PTT and INR. This abnormality notably manifested 24 hours following admission. When grouped by mortality, patients who survived were not coagulopathic by measures of PTT or INR during the 96 hour period (Figure 3). In comparison, patients who died had prolonged periods of coagulopathy that manifested early in their hospital course.

\subsection{Coagulation Factor Activity}

Coagulation factor activity of factors II, V, VII, VIII, IX, X, an XI obtained at admission time zero are summarized in Table 1. At admission, seven patients exhibited increased factor VIII and IX activity beyond normal ranges. Mortality patients had increased mean factor VIII $(600 \pm 1 \%$ vs $283 \pm 134 \%)$ and IX (189 $\pm 14 \%$ vs $158 \pm 19 \%$ ) activity compared to those who survived. Additional abnormalities were seen in factors II, X, and XI at admission, but only in one or two patients each. Admission factor V and VII activity remained within normal range. Grouped by burn size, all patients under $75 \%$ TBSA demonstrated increased factor VIII and IX activity at admission. Of those in the severe burn group, only the two patients who died had elevated factor VIII and IX activity. 
Severely burned patients also demonstrated decreased mean activity compared to the low burn size group in factor II $(75 \pm 8 \%$ vs $66 \pm 18 \%)$, VII $(95 \pm 28 \%$ vs $71 \pm 12 \%)$, VIII $(418 \pm 164 \%$ vs $376 \pm 260 \%)$, IX $(181 \pm 20 \%$ vs $158 \pm 22 \%), X$ ( $73 \pm 12 \%$ vs $65 \pm 19 \%)$, and $\mathrm{XI}(98 \pm 30 \%$ vs $93 \pm 20 \%)$.

Looking over the ensuing 96 hour study period, factor VII was the only coagulation factor whose mean activity remained within normal range and had no major differences between survival groups. Mean factor VIII activity was initially higher in patients who died compared to those who survived. However, this increased activity was only apparent from admission up to the first 24 hours of hospitalization. Afterwards, patients who survived had higher factor VIII activity (Figure 4). This was, however, not statistically significant. Factor IX activity was higher and beyond normal ranges in survival patients compared to mortality patients. This difference was significant at hours 12 and 24 following admission $(p<0.05$, Figure 4).

Grouped by burn size, factor VII activity remained within normal range in all patients in the initial 24 hour period, followed by decreased, below normal activity in four patients by 36 hours. Factor VIII exhibited differential activity. At admission, low and moderately sized burns had elevated factor VIII activity while severe burns were delayed in their elevated response. Additionally, lower sized burns generally maintained this elevated factor VIII activity while moderately sized burns decreased their activity within 24 hours. Factor IX activity was similar to factor VIII activity where lower and moderately sized burns had higher activity 
than severely burned patients at admission. However, maximal factor IX activity was seen in all surviving patients by the 96 hour time point.

\subsection{Anticoagulant Activity}

Natural anticoagulant activity of antithrombin and protein $\mathrm{C}$ obtained at admission time zero are summarized in Table 2. Antithrombin activity was borderline normal or below normal in all patients. Patients who died had increased antithrombin activity compared to patients who survived $(90.8 \pm 6.9 \%$ vs $71.6 \pm 22.6 \%$ ). However, mean antithrombin activity was notably decreased from $90 \pm 6.2 \%$ in the low burn size group to $76.5 \pm 14.9 \%$ in moderate and $74.5 \pm 27.4 \%$ in severe burn groups. Mean antithrombin activity was slightly higher in patients who survived compared to patients who died during the initial 24 hours following admission, but overall remained borderline low or below normal throughout the 96 hour study period. (Figure 5).

Protein C activity at admission was similarly borderline normal or below normal in all patients. Patients who died had decreased mean protein $\mathrm{C}$ activity at admission compared to those who survived ( $54 \pm 20 \%$ vs $78.2 \pm 11.8 \%)$. Mean protein $\mathrm{C}$ activity was below normal in low $(66 \pm 33 \%)$ and moderate sized burns $(62.5 \pm 22 \%)$ and borderline in the severely burned group $(71 \pm 9.6 \%)$. Protein C mean activity also remained borderline low or below normal activity ranges for the ensuing 96 hour study period between survival and burn size groups (Figure 5).

Admission TFPI concentrations were within normal limits and without major differences between survivor groups. However, by hour 12, differences 
between mortality and survivor patients grew larger and continued to increase up to the 96 hour interval. These differences were significant at hours 36 and 60 following admission ( $p<0.05$; Figure 5).

\subsection{Clot Formation and Fibrinolytic Activity}

Concentrations of PAP, D-Dimer, and Fibrin Monomers obtained from plasma at admission time zero are summarized in Table 3. Similar concentrations of PAP at admission were noted between survival groups $(10.3 \pm 6.7 \mathrm{nM}$ vs $9.9 \pm 6.5 \mathrm{nM})$ while differential concentrations were noted between low $(10.3 \pm 6.3 \mathrm{nM})$, moderate $(3.9 \pm 1.4 \mathrm{nM})$ and severe $(13.0 \pm 6.1 \mathrm{nM})$ burn sizes. However, all patients had PAP concentrations beyond normal limits at admission and up to 24 hours after. Mortality and survival patients' PAP concentrations also appeared to trend similarly until hour 60 when they begin to diverge (Figure 6).

Decreased mean concentrations of D-Dimer at admission were noted in patients who died compared to those who survived $(3.5 \pm 3 \mathrm{ug} / \mathrm{mL}$ vs $5.3 \pm 4.2$ $u g / m L)$. This difference was not significant. Lower mean concentrations were also noted in moderate $(1.13 \pm 0.3 \mathrm{ug} / \mathrm{mL})$ and severe burn sizes $(5.01 \pm 3.4$ $\mathrm{ug} / \mathrm{mL})$ compared to low burn sizes $(6.1 \pm 4.3 \mathrm{ug} / \mathrm{mL})$. By hour 8 , mean D-Dimer concentrations remained higher in patients who died than patients who survived up to the 96 hour time point (Figure 6).

Fibrin monomer concentrations obtained from admission plasma were increased in patients who died compared to patients that survived $(107.6 \pm 91.7$ $\mathrm{ug} / \mathrm{mL}$ vs $56.31 \pm 52.2 \mathrm{ug} / \mathrm{mL}$ ). This difference was also not significant. Similar to 
D-Dimer concentrations, decreased fibrin monomer concentrations were noted in moderate $(66.4 \pm 70.2 \mathrm{ug} / \mathrm{mL})$ and severe burn sizes $(74 \pm 46.9 \mathrm{ug} / \mathrm{mL})$ compared to the low burn size group $(94.4 \pm 121.1 \mathrm{ug} / \mathrm{mL})$. Fibrin monomer concentrations also remained elevated and higher in mortality patients than survival patients up to the 96 hour time point (Figure 6).

\subsection{Cytokine Activity}

Cytokine concentrations of IL-1b, IL-6, IL-10, IL-12p.70 and TNF- $\alpha$ obtained from admission time zero are summarized in Table 4. At the time of admission, all patients showed increased IL-6 levels above the normal range (2.5-117 fold) while IL-1b and TNF- $\alpha$ levels were depressed in all patients. IL-10 levels were elevated in 8 of 9 patients (2.4-32 fold). Mortality patients exhibited higher mean cytokine concentrations than survival patients in IL-1b $(1.1 \pm 1.7$ $\mathrm{pg} / \mathrm{mL}$ vs $0.5 \pm 0.3 \mathrm{pg} / \mathrm{mL}), \mathrm{IL}-6(324.9 \pm 568.8 \mathrm{pg} / \mathrm{mL}$ vs $98.2 \pm 78 \mathrm{pg} / \mathrm{mL}), \mathrm{IL}-10$ (168.5 $\pm 139.7 \mathrm{pg} / \mathrm{mL}$ vs $48.3 \pm 29.6 \mathrm{pg} / \mathrm{mL}), \mathrm{IL}-12 \mathrm{p} .70(4.3 \pm 7 \mathrm{pg} / \mathrm{mL}$ vs $1.5 \pm 1.2$ $\mathrm{pg} / \mathrm{mL})$, and TNF- $\alpha(6.3 \pm 3.2 \mathrm{pg} / \mathrm{mL}$ vs $5.3 \pm 1 \mathrm{pg} / \mathrm{mL})$. These differences, however, are not statistically significant. Burn size related increases in mean cytokine concentration at admission were also observed in $\mathrm{IL}-1 \mathrm{~b}(0.3 \pm 0.3 \mathrm{pg} / \mathrm{mL}$ low vs $0.65 \pm 0.1 \mathrm{pg} / \mathrm{mL}$ moderate vs $1.2 \pm 1.7 \mathrm{pg} / \mathrm{mL}$ severe), IL-6 $(78.4 \pm 79.8 \mathrm{pg} / \mathrm{mL}$ low vs $127.3 \pm 93.1 \mathrm{pg} / \mathrm{mL}$ moderate vs $325 \pm 568.7$ severe $), \mathrm{IL}-10(21.1 \pm 17.1 \mathrm{pg} / \mathrm{mL}$ low vs $169.7 \pm 98.8 \mathrm{pg} / \mathrm{mL}$ moderate vs $128.1 \pm 132$ severe), IL-12p.70 (1.18 \pm 1 $\mathrm{pg} / \mathrm{mL}$ low vs $2.1 \pm 1.4 \mathrm{pg} / \mathrm{mL}$ moderate vs $4.2 \pm 7.1$ severe), and TNF- $\alpha(5.4 \pm 1.4$ $\mathrm{pg} / \mathrm{mL}$ low vs $4.7 \pm 0.7 \mathrm{pg} / \mathrm{mL}$ moderate vs $6.6 \pm 3$ severe). Significant increases from baseline are noted only in mortality patients from 24 hours on in IL-1b, IL-6, 
and IL-12p.70 and from 48 hours on in TNF- $\alpha$ (p<0.05; Figure 7). Interestingly, mortality patients did not mount an increased response with IL-10 until the 96 hour time point. 


\section{Discussion}

Studying the hematologic profile of 102 patients with a burn injury of at least $15 \%$ TBSA or greater, Lu and colleagues found no evidence of coagulation abnormalities by measures of INR, PTT, platelet count, or hemoglobin level within 12 hours of admission [19]. Sherren and colleagues comparatively looked at 117 patients with a burn injury of at least $30 \%$ TBSA or greater shortly after admission and noted $71.7 \%$ of patients with an elevated PT, $2.2 \%$ with an elevated PTT, and $26.1 \%$ with an elevation of both. They estimated that burn induced coagulopathy was an independent predictor of 28 day mortality [12]. Mitra and colleagues looked at 99 patients with a burn injury of at least $20 \%$ TBSA or greater and noted only three patients who presented with a coagulopathy by measures of PTT and INR, but $37(37 \%)$ developed a coagulopathy within 24 hours of admission [7]. In our cohort, no patients were identified with coagulation abnormalities within 24 hours of admission by measures of PTT or INR and only two patients were identified with coagulation abnormalities thereafter. This serves to highlight the limited and conflicting data available on thermally induced coagulation abnormalities. Studies such as these rely on routine measures of coagulation, infrequent measurements, and retrospective data. Coagulation is a rapid and dynamic process whose abnormalities may be short lived and missed using current study methodology.

Relative to previous studies, this study methodology comprising established standardized and frequent blood draws is unique and allows one to prospectively identify immediate and minute changes in coagulation. To our 
knowledge, no study to date has looked at thermally induced coagulation changes with the degree of resolution that our methodology provides. Dynamic changes were seen in clotting factor, anticoagulant, and fibrinolytic behavior. These were noted at admission and throughout the study's time course. Although we identified some changes in coagulation by PTT and INR, these changes lagged behind our factor assays. This, coupled with the inability of PTT and INR to identify hypercoagulable states, underscores some of the problems in using these tests in the setting of acute injury.

Patients who died had worse clinical presentations than those who lived. Patients who died required increased transfusion requirements, mechanical ventilation, and critical care that was reflected in their higher mortality scores. The Baux score incorporates age and burn size to approximate the likelihood of mortality and futility of care, which was $100 \%$ in Baux's original article, but has since increased due to advances in clinical care $[20,21]$. While patients in both groups had high mortality scores, patients who died had higher Baux and FLAMES scores. Additionally, patients who died presented with worse and longer periods of acidosis. Coupled with decreased core body temperatures within the first 24 hours, patients who died were more susceptible to the lethal triad than those who survived.

Factor VII's interaction with tissue factor starts the extrinsic pathway of coagulation and is known to increase in both the trauma and septic populations. Inflammatory cytokines cause increased tissue factor expression on monocytes and associated microparticles that can further drive this process [22, 23]. In our 
cohort, normal factor VII activity was noted at admission in all patients, despite the significantly increased cytokine activity seen in mortality patients compared to baseline.

Factor VIII is a procoagulant involved in factor $\mathrm{X}$ activation and an acute phase reactant. The protein $\mathrm{C}$ mediated pathway thought to drive acute traumatic coagulopathy leads to a decrease in factor VIII activation that may initially place trauma patients in a hypocoagulable state [22, 24]. Septic populations comparatively are thought to have deactivated protein $\mathrm{C}$, leading to increased factor VIII and an overall procoagulant state [23]. Most of our thermally injured cohort in turn had elevated activity of factor VIII. Patients that died had increased factor VIII activity compared to patients that died. Additionally, lower and moderately sized burns had markedly elevated factor VIII activity while severely burned patients who survived did not manifest as high of a response at admission. Interestingly, several other factors were notably decreased in the severe burn group compared to the lower burn size group. This suggests a size dependent response to injury that may affect the coagulation process.

Natural anticoagulant activity has been described with decreased function in both the trauma and septic populations $[22,23]$. Though protein $C$ is notably activated through the interaction of thrombomodulin and thrombin in a shock state, stores eventually deplete and leave the patient in a hypercoagulable state [22]. Our cohort of thermally injured patients seems to reflect this. Despite burn size or mortality, antithrombin and protein $C$ activity was universally low or borderline low in all patients. In the context of increased clotting factor activity, 
this is significant. The decreased anticoagulant activity coupled with increased clotting factor activity may place patients in a hypercoagulable state at admission and throughout their hospital course.

Increased concentrations of PAP, D-Dimer, and fibrin monomers were seen at admission and throughout the 96 hour study period. In the case of DDimer and fibrin monomers, these concentrations remained elevated in mortality patients compared to survivor patients. The presence of elevated D-Dimer and fibrin monomer concentrations suggest increased clot formation and, along with the clotting factor and anticoagulant findings, further support a hypercoagulable status for the patients included in this study. The elevated PAP levels at admission imply increased fibrinolytic activity in response to this hypercoabulable state. Note that the decreasing PAP concentration trend in the subsequent 96 hours may be showing an attenuation of the fibrinolytic response.

Time zero admission data was additionally reviewed to identify trends and behaviors. Overall there is increased activity of factor VII and IX in most patients with a concomitant decreased activity in natural anticoagulants antithrombin III and protein $\mathrm{C}$ compared to normal ranges. A concomitant hyperfibrinolytic response is also seen as exhibited by elevated PAP, D-Dimer, and fibrin monomer concentrations at admission. These data suggest that thermally injured patients may already present in a hypercoagulable state at admission. Identification of these states during the pretreatment phase of burn injury could provide an early time point for therapeutic intervention that may affect survival. 
Sample collection anticoagulant tubes, or SCAT, were used in this study to provide blood sample preservation and allow later testing in a variety of nonroutine blood tests. A broad range of anticoagulant and proteinase inhibitors are used to inhibit the broad range of proteolytic enzymes potentially released during burn related injury. This allowed for blood sample stabilization and subsequent unaltered analysis.

There are several limitations of this study. Though this cohort provides a snapshot of the minute and transient changes in coagulation seen in thermal injury, this is a single center study with a small sample size. A higher powered study is required to make any definitive statements on behavior or mortality. As previously mentioned, routine measures of coagulation have limitations while other more advanced coagulation tests, such as thromboelastography, could provide further insight into clotting behavior and characteristics. Additionally, routine laboratory studies were obtained at the discretion of the managing medical team and were therefore not obtained daily compared to standardized blood draws. Obtaining PTT and INR concomitantly at standardized blood draws could allow a more comprehensive comparison of hematologic profiles. Finally, additional clinical parameters, such as fluid or operative management, could provide further characterization of coagulation behavior in the context of clinical management. 


\section{Conclusion}

There are complex and dynamic changes in coagulation, anticoagulation, and fibrinolysis following thermally injury that may not detected by routine measures of coagulation alone. Though abnormal coagulation has been identified in this cohort, it remains poorly characterized in the thermally injured population. This novel approach further characterizes the dynamic changes in coagulation following thermal injury and potentially identifies mechanisms that might suggest therapeutic intervention. 
Acknowledgements: The authors thank Stago for their in-kind support of assays.

Author Contributions: All authors have made equal contributions to the conception, design, analysis, interpretation, drafting, and revision of the manuscript for this study. 


\section{References}

1. Brohi, K., et al., Acute traumatic coagulopathy. J Trauma, 2003. 54(6): p. 1127-30.

2. Brohi, K., et al., Acute coagulopathy of trauma: hypoperfusion induces systemic anticoagulation and hyperfibrinolysis. J Trauma, 2008. 64(5): p. 1211-7; discussion 1217.

3. MacLeod, J.B., et al., Early coagulopathy predicts mortality in trauma. J Trauma, 2003. 55(1): p. 39-44.

4. Frith, D., et al., Definition and drivers of acute traumatic coagulopathy: clinical and experimental investigations. J Thromb Haemost, 2010. 8(9): p. 1919-25.

5. Valparaiso, A.P., et al., Modeling acute traumatic injury. J Surg Res, 2015. 194(1): p. 220-232.

6. Cap, A. and B. Hunt, Acute traumatic coagulopathy. Curr Opin Crit Care, 2014. 20(6): p. 638-45.

7. Mitra, B., et al., Early coagulopathy of major burns. Injury, 2013. 44(1): p. 40-3.

8. Van Haren, R.M., et al., Hypercoagulability after burn injury. J Trauma Acute Care Surg, 2013. 75(1): p. 37-43; discussion 43.

9. Garcia-Avello, A., et al., Degree of hypercoagulability and hyperfibrinolysis is related to organ failure and prognosis after burn trauma. Thromb Res, 1998. 89(2): p. 59-64.

10. Jurkovich, G.J. and J.A. Moylan, Inhalation injury--a major burn complication. Physician Assist, 1983. 7(10): p. 59-62, 65-8.

11. King, D.R., N. Namias, and D.M. Andrews, Coagulation abnormalities following thermal injury. Blood Coagul Fibrinolysis, 2010. 21(7): p. 666-9.

12. Sherren, P.B., et al., Acute burn induced coagulopathy. Burns, 2013. 39(6): p. 1157-61.

13. Lavrentieva, A., Replacement of specific coagulation factors in patients with burn: a review. Burns, 2013. 39(4): p. 543-8.

14. Park, M.S., et al., Thromboelastography as a better indicator of hypercoagulable state after injury than prothrombin time or activated partial thromboplastin time. J Trauma, 2009. 67(2): p. 266-75; discussion 275-6.

15. Fecher, A.M., et al., Analysis of deep vein thrombosis in burn patients. Burns, 2004. 30(6): p. 591-3.

16. Pannucci, C.J., et al., Acquired inpatient risk factors for venous thromboembolism after thermal injury. J Burn Care Res, 2012. 33(1): p. 84-8.

17. Korte, W. and L. Graf, Burn injuries: is antithrombin back on stage in critical care? Thromb Haemost, 2008. 100(2): p. 177-8.

18. Gomez, M., et al., The FLAMES score accurately predicts mortality risk in burn patients. J Trauma, 2008. 65(3): p. 636-45.

19. Lu, R.P., et al., Major burn injury is not associated with acute traumatic coagulopathy. J Trauma Acute Care Surg, 2013. 74(6): p. 1474-9. 
20. Roberts, G., et al., The Baux score is dead. Long live the Baux score: a 27-year retrospective cohort study of mortality at a regional burns service. J Trauma Acute Care Surg, 2012. 72(1): p. 251-6.

21. Jeschke, M.G., et al., Sex differences in the long-term outcome after a severe thermal injury. Shock, 2007. 27(5): p. 461-5.

22. Maegele, M., H. Schochl, and M.J. Cohen, An update on the coagulopathy of trauma. Shock, 2014. 41 Suppl 1: p. 21-5.

23. Simmons, J. and J.F. Pittet, The coagulopathy of acute sepsis. Curr Opin Anaesthesiol, 2015.

24. Noel, P., S. Cashen, and B. Patel, Trauma-induced coagulopathy: from biology to therapy. Semin Hematol, 2013. 50(3): p. 259-69. 


\section{Figure Captions}

Figure 1: Baux and FLAMES mortality scores showing significantly higher mortality scores in patients who died compared to those who lived.

Figure 2: Clinical laboratory measures of acidosis by blood $\mathrm{pH}$, serum $\mathrm{CO} 2$, and base change grouped by survival and compared to normal laboratory ranges. Each box indicates one time point. Note prolonged measures of acidosis in patients who died in all three measures.

Figure 3: Clinical laboratory measures of coagulation by measures of PTT and INR. PTT and INR values are grouped by survival and compared to normal laboratory measures. Each box indicates one time point. Patients who survived had no abnormal measures of PTT or INR.

Figure 4: Factor VII, VIII, and IX activity over the 96 hour study period. Activity values are grouped by survival and compared for trends and significance. Dashed lines represent the lower and upper limits of the normal range. * denotes significance $(p<0.05)$ with the associated solid line indicating the time points being compared.

Figure 5: Antithrombin and protein C activity as well as TFPI concentration over the 96 hour study period. Activity values and concentrations are grouped by survival and compared for trends and significance. Dashed lines represent the 
lower and upper limits of the normal range. ${ }^{*}$ denotes significance $(p<0.05)$ with the associated solid line indicating the time points being compared.

Figure 6: PAP, D-Dimer, and fibrin monomer concentration over the 96 hour study period. Concentrations are grouped by survival and compared for trends and significance. Dashed lines represent the lower and upper limits of the normal range. ${ }^{*}$ denotes significance $(p<0.05)$ with the associated solid line indicating the time points being compared. (Normal PAP $<6 \mathrm{nM}$, normal D-Dimer $<0.5$ $\mu \mathrm{g} / \mathrm{mL}$, normal fibrin monomer $<6 \mu \mathrm{g} / \mathrm{mL}$ )

Figure 7: The percent change in concentration of cytokines IL-1b, IL-6, IL-10, IL12p.70, and TNF- $\alpha$ over the 96 hour study period compared to baseline time zero results. Concentrations are grouped by survival and compared for trends and significance. Dashed lines represent the lower and upper limits of the normal range. ${ }^{*}$ denotes significance $(p<0.05)$ with the associated solid line indicating the time points being compared. 


\section{Table Captions}

Table 1: Factor activity obtained from plasma samples at admission time zero and compared to normal ranges. * denotes patient death. Red highlighted values indicate activity levels out of the normal range. TBSA scores: low - blue, moderate - yellow, and severe - gray.

Table 2: Anticoagulant activity obtained from plasma samples at admission time zero and compared to normal ranges. * denotes patient death. Red highlighted values activity levels out of the normal range. TBSA scores: low - blue, moderate - yellow, and severe - gray.

Table 3: Fibrinolytic and clot formation marker concentrations obtained from plasma samples at admission time zero. Values are compared to normal ranges to produce a fold change from normal. ${ }^{*}$ denotes patient death. Fold change is reported as it relates to the upper limit of the normal range. TBSA scores: low blue, moderate - yellow, and severe - gray.

Table 4: Cytokine concentrations obtained from plasma samples at admission time zero. Values are compared to normal ranges to produce a fold change from normal. Note that IL-12p.70 has no defined normal range. * denotes patient death. Fold change is reported as it relates to the upper limit of the normal range. TBSA scores: low - blue, moderate - yellow, and severe - gray. 
Table 1: Coagulation Factor Levels at Admission

\begin{tabular}{|c|c|c|c|c|c|c|c|c|}
\hline \multicolumn{10}{|c|}{ Factor Activity (\%) } \\
\hline Pt & TBSA & II & V & VII & VIII & IX & X & XI \\
\hline 1 & 25 & 80 & 121 & 119 & 371 & 160 & 72 & 109 \\
2 & $26.5^{*}$ & 66 & 95 & 103 & 600 & 200 & 62 & 64 \\
3 & 36 & 78 & 65 & 64 & 282 & 182 & 85 & 121 \\
4 & 50 & 95 & 126 & 124 & 457 & 171 & 91 & 110 \\
5 & $60^{*}$ & 102 & 99 & 71 & 599 & 200 & 100 & 200 \\
6 & $80^{*}$ & 86 & 99 & 81 & 600 & 180 & 71 & 121 \\
7 & 80 & 55 & 84 & 57 & 112 & 133 & 61 & 82 \\
8 & 90 & 47 & 68 & 64 & 192 & 146 & 41 & 94 \\
9 & $95^{*}$ & 77 & 138 & 80 & 600 & 174 & 87 & 75 \\
\hline
\end{tabular}

* Patient mortality

Table 2: Anticoagulant Factor Levels at Admission

\begin{tabular}{|c|c|c|c|c|}
\hline \multicolumn{5}{|c|}{ Anticoagulant Activity (\%) } \\
\hline Pt & TBSA & $\begin{array}{c}\text { ATIII } \\
\text { (\%) }\end{array}$ & $\begin{array}{c}\text { PC } \\
\text { (\%) }\end{array}$ & $\begin{array}{c}\text { TFPI } \\
\text { (nM) }\end{array}$ \\
\hline 1 & 25 & 95 & 73 & 2 \\
2 & $26.5^{\star}$ & 83 & 30 & 1.9 \\
3 & 36 & 92 & 95 & 2.1 \\
4 & 50 & 66 & 78 & 3.4 \\
5 & $60^{*}$ & 87 & 47 & 3.1 \\
6 & $80^{*}$ & 96 & 63 & 1.7 \\
7 & 80 & 65 & 82 & 1.8 \\
8 & 90 & 40 & 63 & 1.9 \\
9 & $95^{*}$ & 97 & 76 & 2 \\
\hline
\end{tabular}

* Patient mortality 


\section{Table 3: Markers of Fibrin Lysis and Formation at Admission}

\begin{tabular}{|c|c|c|c|c|c|c|c|}
\hline \multicolumn{9}{|c|}{ Clot Formation/Fibrinolytic Markers } \\
\hline \multicolumn{2}{|c|}{} & \multicolumn{2}{|c|}{ PAP } & \multicolumn{2}{c|}{ D-Dimer } & \multicolumn{2}{c|}{$\begin{array}{c}\text { Fibrin } \\
\text { Monomer }\end{array}$} \\
\hline Pt & TBSA & $\mathrm{nM}$ & Fold $\Delta$ & $\mu \mathrm{g} / \mathrm{mL}$ & Fold $\Delta$ & $\mu \mathrm{g} / \mathrm{mL}$ & Fold $\Delta$ \\
\hline 1 & 25 & 3.9 & 0.65 & 1.16 & 2.32 & 20.58 & 3.43 \\
2 & $26.5^{\star}$ & 10.43 & 1.74 & 7.7 & 15.4 & 234.15 & 39.03 \\
3 & 36 & 16.5 & 2.75 & 9.3 & 18.6 & 28.6 & 4.77 \\
4 & 50 & 2.97 & 0.5 & 1.32 & 2.64 & 16.77 & 2.8 \\
5 & $60^{*}$ & 4.88 & 0.81 & 0.94 & 1.88 & 116 & 19.3 \\
6 & $80^{*}$ & 6.08 & 1.01 & 1.8 & 3.6 & 44.01 & 7.34 \\
7 & 80 & 9.84 & 1.64 & 4.7 & 9.4 & 139.3 & 23.2 \\
8 & 90 & 16.26 & 2.71 & 9.85 & 19.7 & 76.3 & 12.7 \\
9 & $95^{*}$ & 19.7 & 3.28 & 3.69 & 7.38 & 36.35 & 6.06 \\
\hline
\end{tabular}

${ }^{*}$ Patient mortality

${ }^{* *}$ Fold $\Delta$ as compared to its upper limit of normal

Table 4: Markers of Inflammation at Admission

\begin{tabular}{|c|c|c|c|c|c|c|c|c|c|c|c|}
\hline \multicolumn{10}{|c|}{ Cytokine Data } \\
\hline Pt & TBSA & pg/mL & Fold $\Delta$ & pg/mL & Fold $\Delta$ & pg/mL & Fold $\Delta$ & pg/mL & Fold $\Delta$ & pg/mL & Fold $\Delta$ \\
\hline 1 & 25 & 0.62 & 0.62 & 171 & 17.1 & 36.6 & 3.7 & 2.32 & & 6.89 & 0.442 \\
2 & $26.5^{*}$ & 0.13 & 0.13 & 34.6 & 3.5 & 2.76 & 0.28 & 0.62 & & 4.17 & 0.267 \\
3 & 36 & 0.19 & 0.185 & 30 & 3 & 24 & 2.4 & 0.61 & & 5.02 & 0.322 \\
4 & 50 & 0.72 & 0.717 & 193 & 19.3 & 99.8 & 10 & 3.16 & & 5.14 & 0.329 \\
5 & $60^{*}$ & 0.58 & 0.58 & 61.4 & 6.1 & 240 & 24 & 1.13 & & 4.21 & 0.27 \\
6 & $80^{*}$ & 0.12 & 0.12 & 25.4 & 2.5 & 112 & 11.2 & 0.75 & & 6.05 & 0.388 \\
7 & 80 & 0.72 & 0.72 & 64.5 & 6.5 & 42 & 4.2 & 0.71 & 4.14 & 0.265 \\
8 & 90 & 0.29 & 0.292 & 32.3 & 3.2 & 38.9 & 3.9 & 0.6 & & 5.04 & 0.323 \\
9 & $95^{*}$ & 3.72 & 3.72 & 1178 & 117 & 320 & 32 & 14.8 & 11 & 0.703 \\
\hline
\end{tabular}

* Patient mortality

${ }^{* *}$ Fold $\Delta$ as compared to its upper limit of normal 

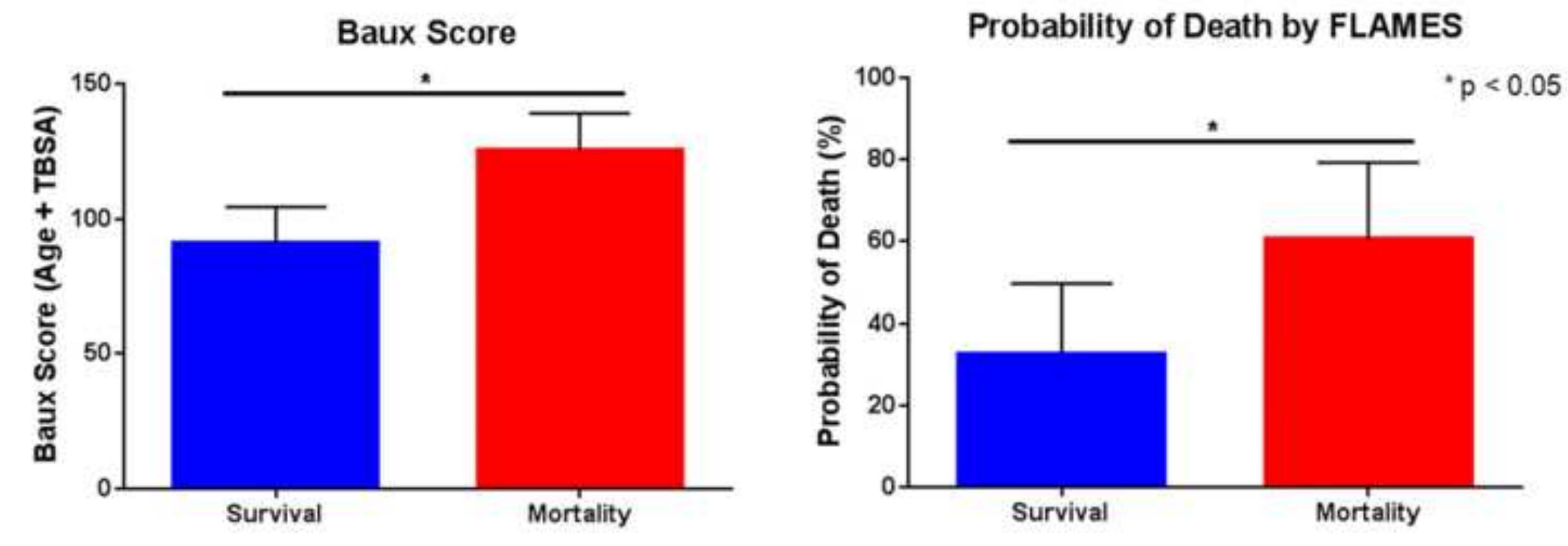
pH By Mortality

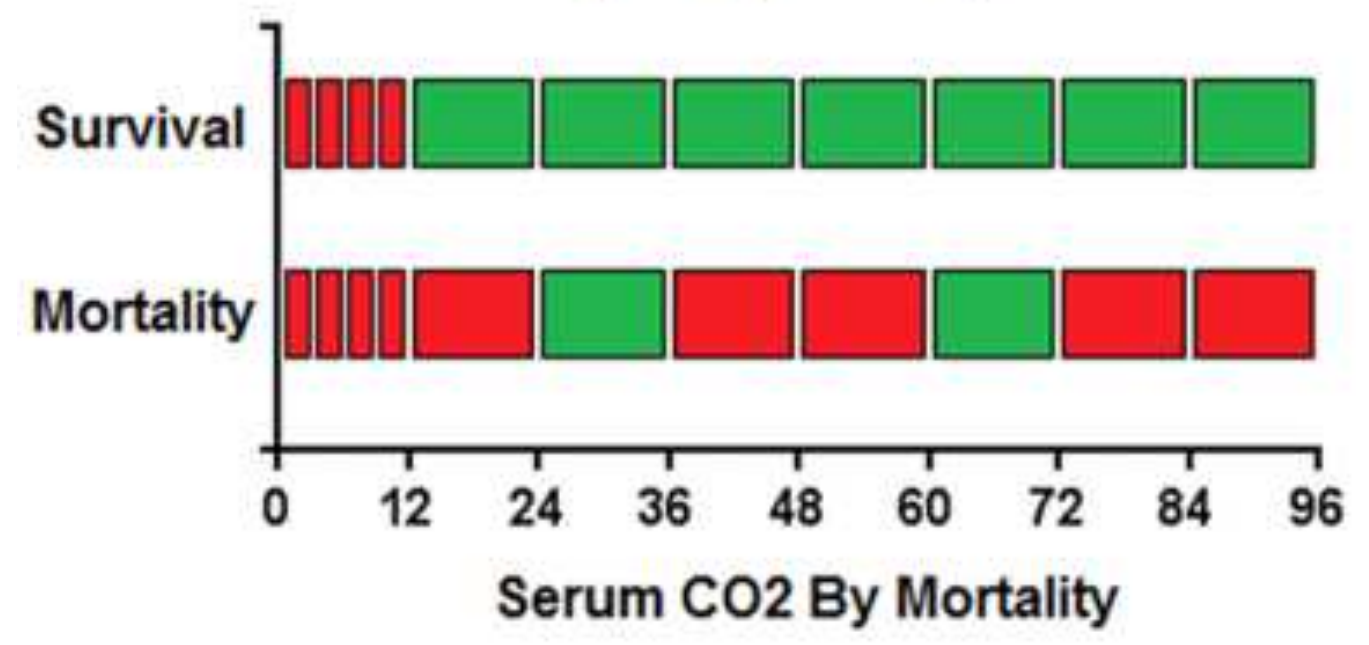

$\square$ Normal Range

$\square$ Acidotic $(\mathrm{pH}<7.35)$

$\square$ Alkalotic $(\mathrm{pH}>7.45)$

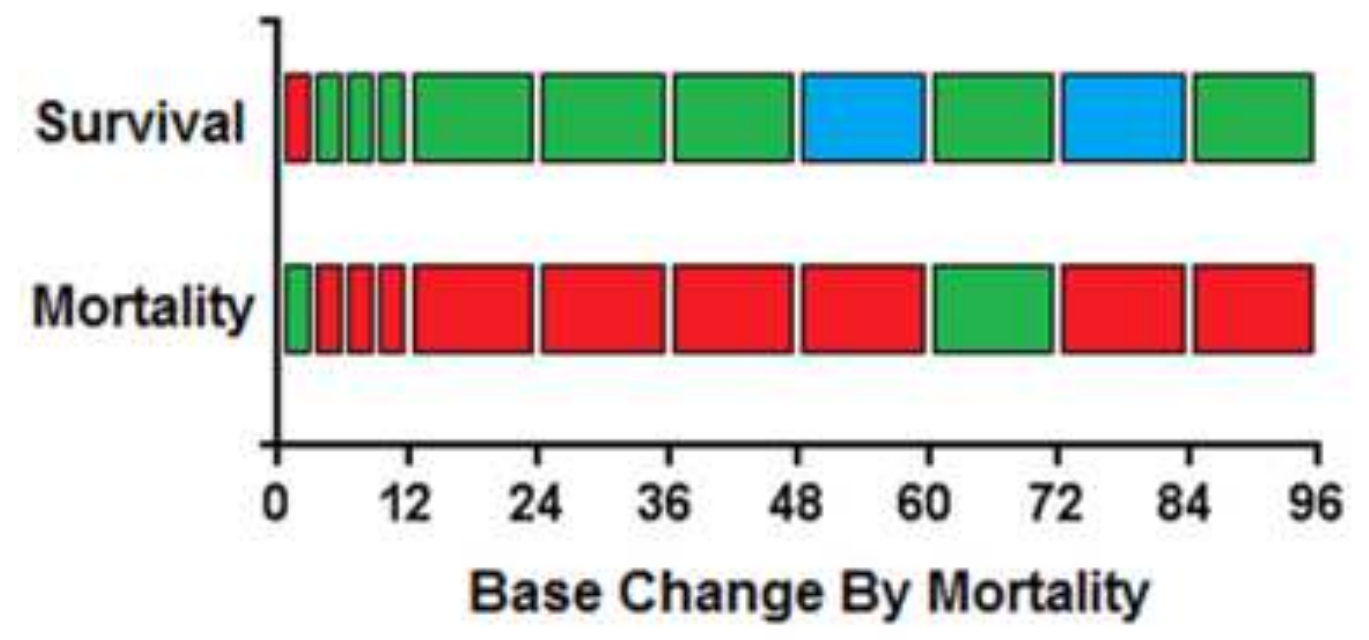

$\square$ Normal Range

$\square$ Below Normal (<22 mEq/L)

$\square$ Above Normal (> $28 \mathrm{mEq} / \mathrm{L}$ )

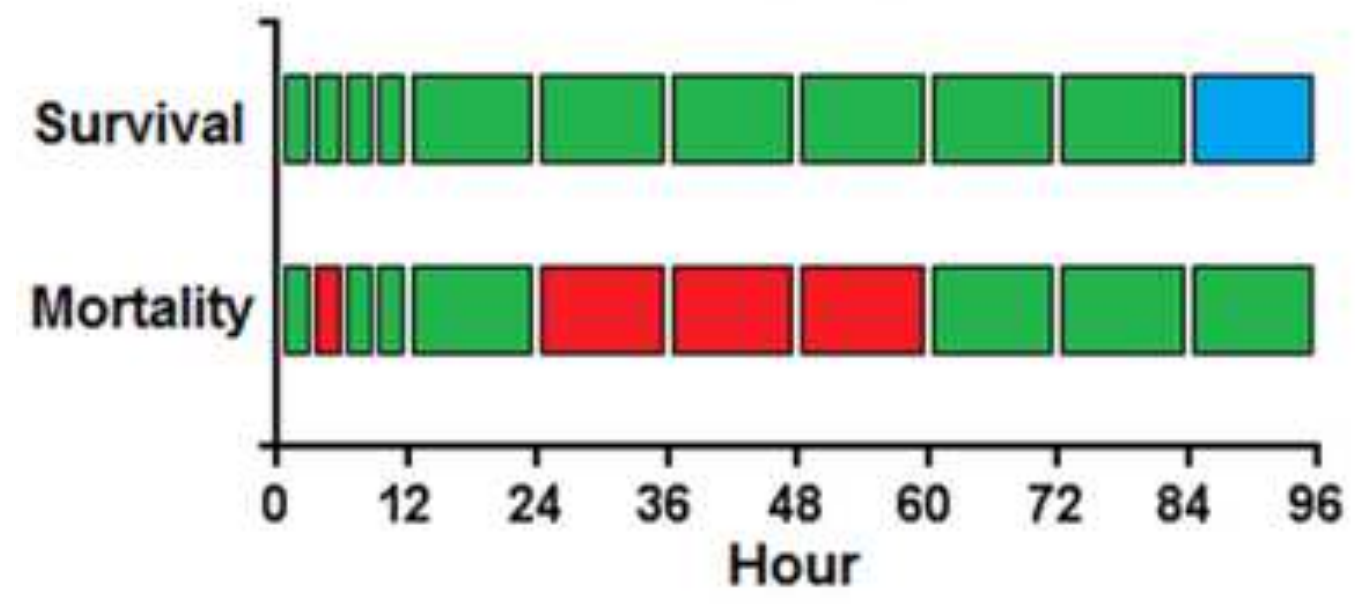

$\square$ Normal Range

$\square$ Base Deficit (<-2 mEq/L)

$\square$ Base Excess $(>+2 \mathrm{mEq} / \mathrm{L})$ 
Coagulation Status By INR

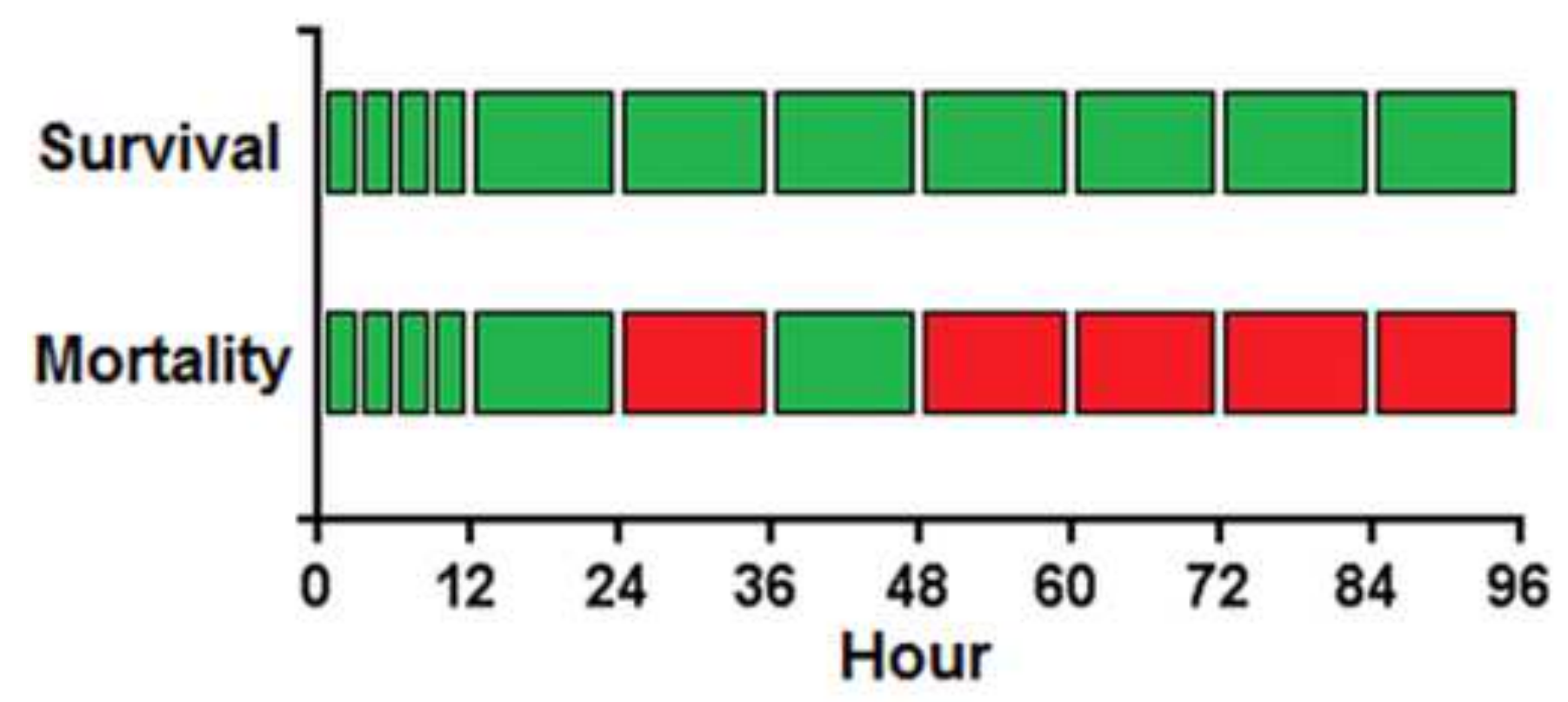

$\square$ Normal

$\square$ Coagulopathy (INR > 1.5)

Coagulation Status by PTT

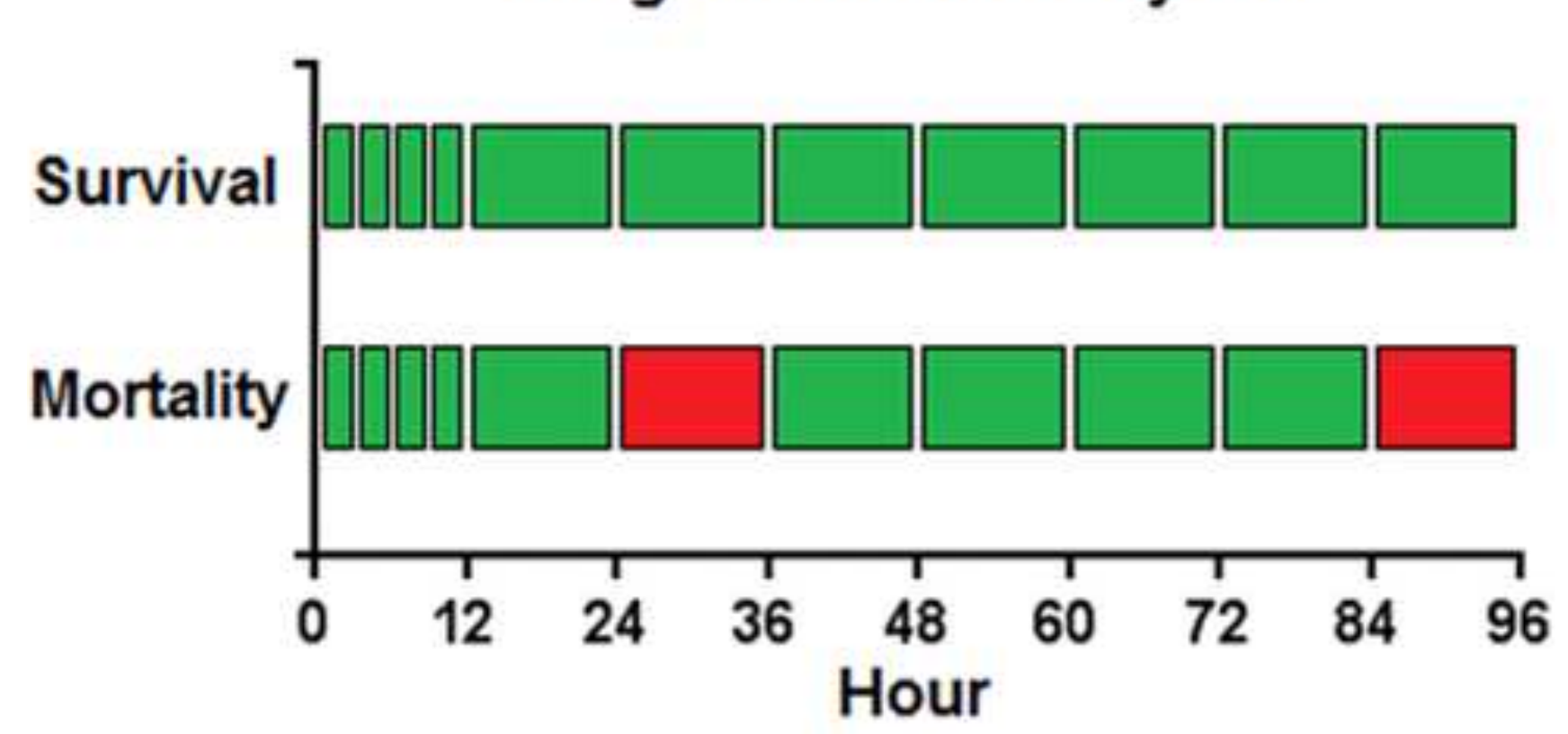

$\square$ Normal

$\square$ Coagulopathy (PTT > 45)

\section{Hour}



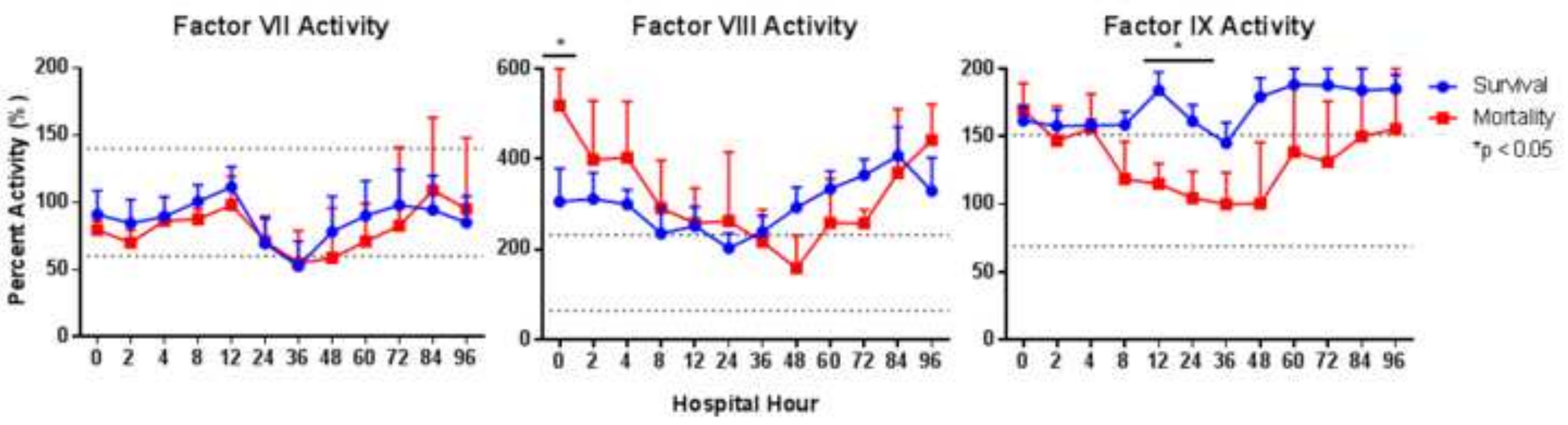


\section{Figure 5}
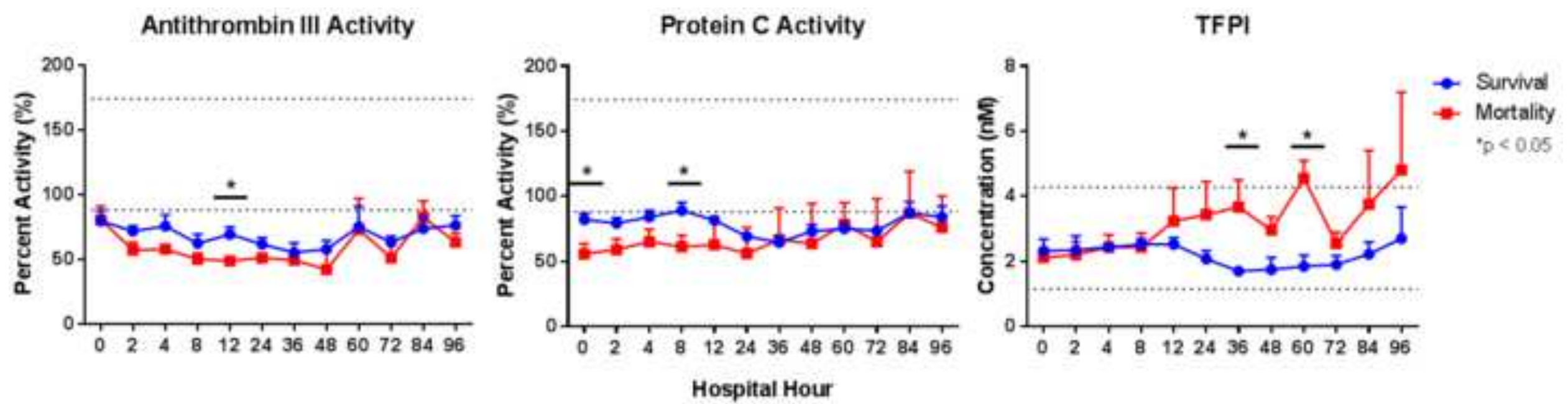

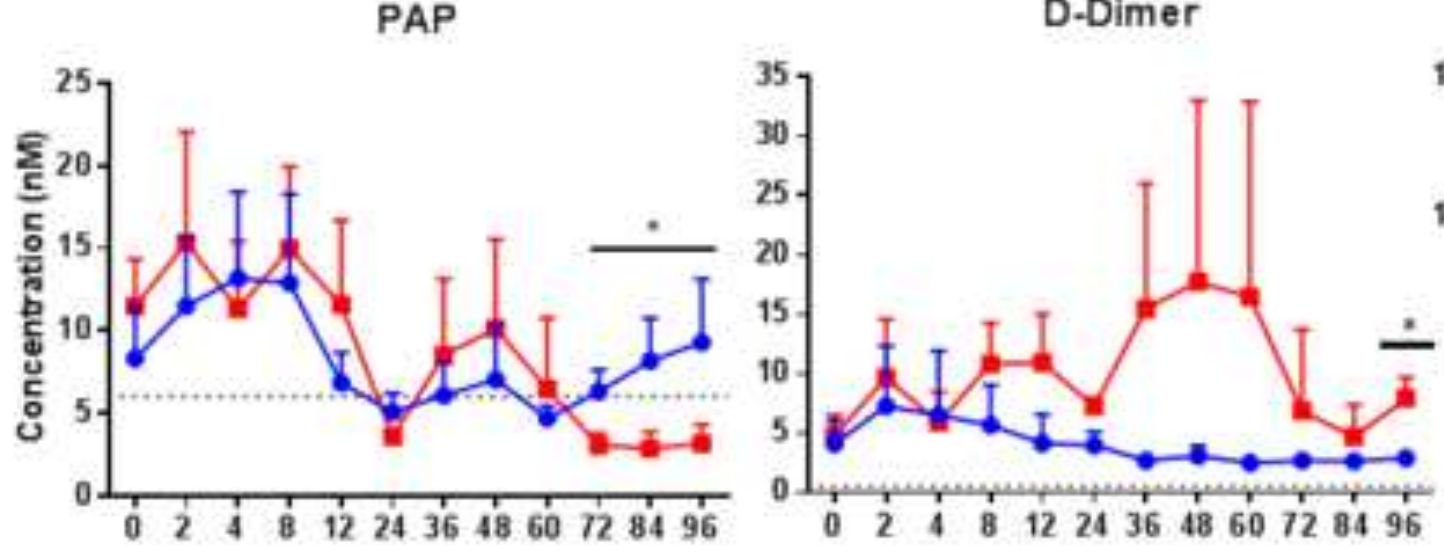

Fibrin Monomers

Hospital Hour 
IL-1

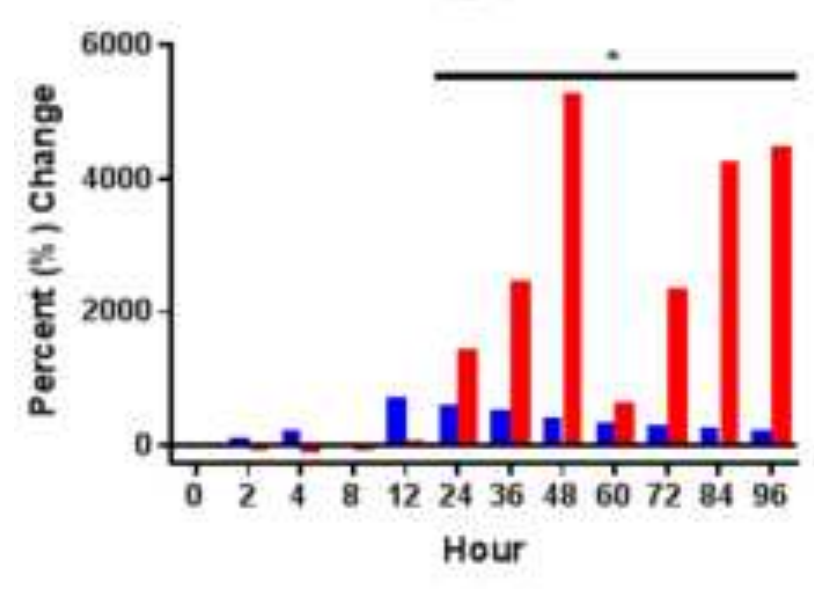

IL-12

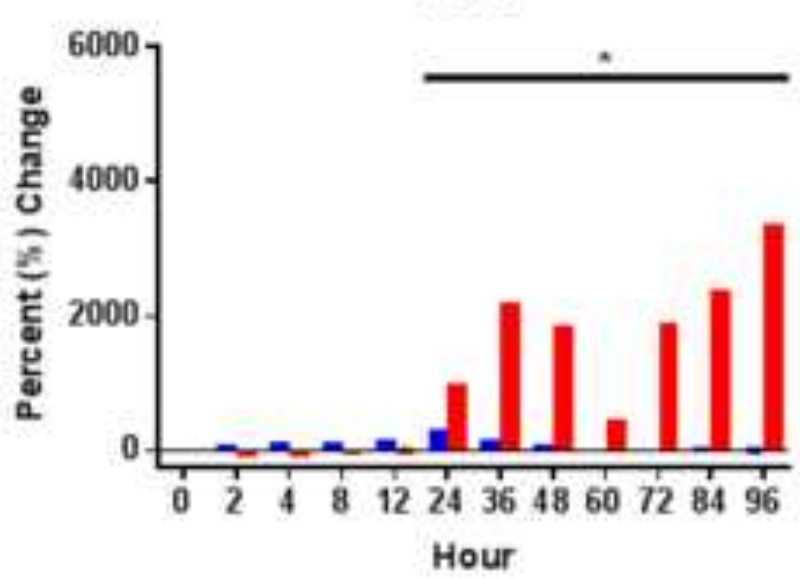

IL- 6

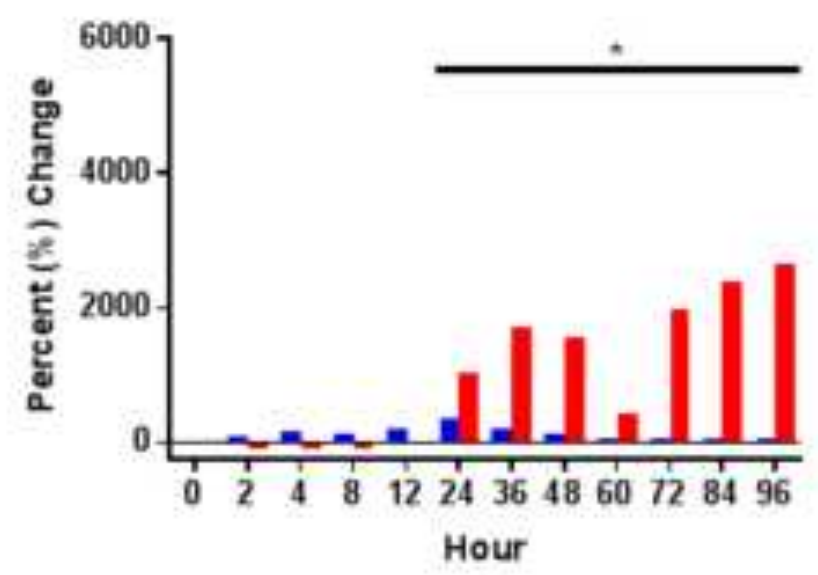

TNF- $\alpha$

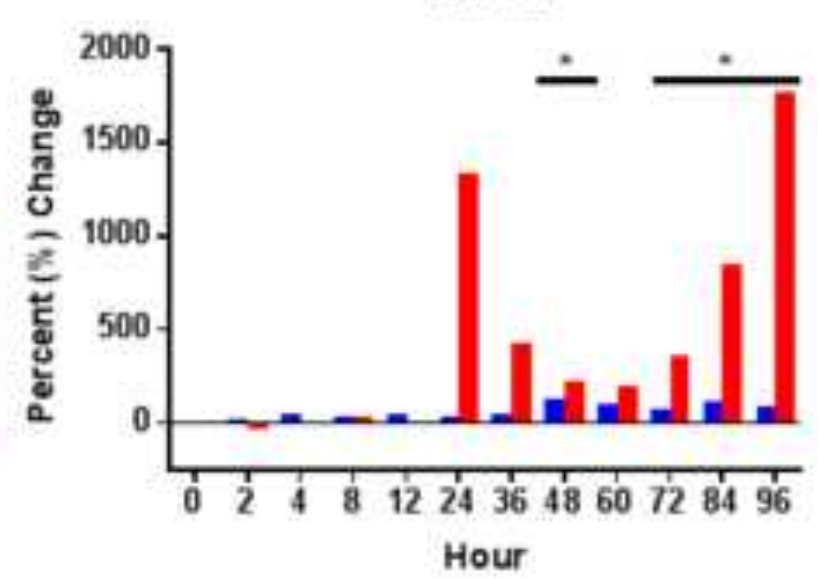

IL-10

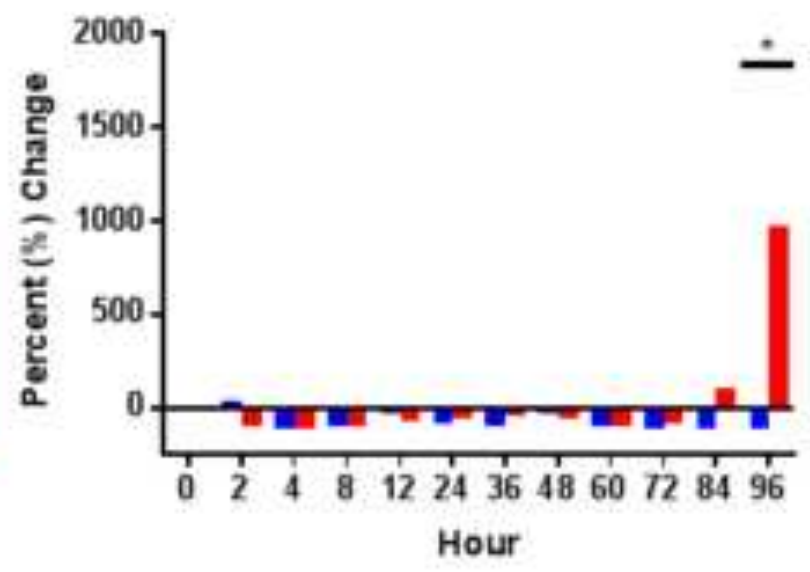

- Survial

- Mortality " $p<0.05$ 